\title{
Comparative Analysis of Vertical Transmission of SARS-CoV-2 Antibodies in Vaccinated and Non-Vaccinated Pregnant Women
}

\author{
Madalina IORDACHE ${ }^{1}$, Diana SECARA ${ }^{1}$, Claudia MEHEDINTU ${ }^{2}$, Marina ANTONOVICl ${ }^{2}$, Natalia TURCAN ${ }^{3}$, \\ Monica Mihaela CIRSTOIU ${ }^{1}$
}

\begin{abstract}
Pregnancy is a risk factor for developing a severe, complicated form of COVID-19. Medical reports have revealed that pregnancy increases three times the risk of ICU admission and 1.7 times the risk of death in patients with COVID-19. The crossing of the placenta by the antibodies generated through vaccination offer a level of protection that should not be ignored. We aimed to comparatively analyze the levels of SARS-CoV-2 IgG and IgM antibodies in pregnant women who have had this infection during pregnancy or have undergone a complete vaccination cycle during pregnancy, as well as antibody levels in newborns. The inclusion criterion was history of SARS-CoV-2 infection during pregnancy or COVID-19 complete vaccination. For each case the peri-partum values of IgG and IgM SARSCoV-2 antibodies were analyzed in the same laboratory along with those of their newborns. The vaccination rate in our study group was about $6 \%$. All cases had a significant value of protective IgG SARS-CoV-2 antibodies and the level of protective antibodies of the newborns closely followed maternal values. From the cases with SARS-CoV-2 infection during pregnancy, only $16.6 \%$ had a protective level of antibodies and $75 \%$ of the newborns from these cases had protective levels of IgG SARS-CoV-2 antibodies. Our results clearly plead in favor of vaccination in pregnancy which provides significant benefits for both mothers and infants.
\end{abstract}

Keywords: SARS-CoV-2, COVID-19, vaccination, pregnancy.

'Department of Obstetrics and Gynecology, „Carol Davila” University of Medicine and Pharmacy, Emergency University Hospital, Bucharest, Romania

2Department of Obstetrics and Gynecology, „Nicolae Malaxa”

Clinical Hospital, "Carol Davila" University of Medicine and

Pharmacy, Bucharest, Romania

${ }^{3}$ Department of Obstetrics and Gynecology, Emergency University

Hospital, Bucharest, Romania

\section{Corresponding author.}

Natalia TURCAN, Department of Obstetrics and Gynecology, Emergency University Hospital, Bucharest, Romania

E-mail: napritcan@gmail.com 


\section{INTRODUCTION}

COVID-19 pandemic has had a significant impact on all previously ordinary activities and effectively changed our lives. It is now known that pregnancy is a risk factor for developing a severe, complicated form of COVID-19; yet, as the records show so far, only $22 \%$ of pregnant women have received at least one dose of COVID-19 vaccine in the United States, where the overall vaccination rate is among the highest in the world ${ }^{1}$. Medical reports have revealed that pregnancy increases three times, the risk of ICU admission and 1.7 times, the risk of death in the context of COVID-19, compared to non-pregnant women. Currently, comorbidities such as obesity, hypertension and diabetes have a rising incidence among the pregnant population, along with a growing maternal age. All these factors increase the risk of an unfavorable outcome ${ }^{3}$. Although the impact of SARS-CoV-2 (Severe Acute Respiratory Syndrome Coronavirus 2) infection on obstetric outcome is still being investigated, a causal relationship with preterm birth, cesarean delivery and fetal distress has been highlighted by studies to date. ${ }^{4}$.

Vaccination is seen as the only way to regain normalcy in our lives. When the first COVID-19 vaccine was approved, the status of pregnant woman was a relative contraindication, but now, most specialized associations openly plead for vaccination during pregnancy.

The American College of Obstetricians and Gynecologists (ACOG) along with the Society for Maternal-Fetal Medicine (SMFM), two reference organizations, support vaccination during pregnancy arguing that COVID-19 vaccine is safe and the lack of the protection the vaccine could provide is now visible in countries with low vaccination rates ${ }^{1}$.

The Royal College of Obstetricians and Gynecologists also recommends COVID-19 vaccine to all pregnant women aged 18 and over, stating that COVID-19 vaccine does not contain ingredients that could have a detrimental effect on pregnancy ${ }^{5}$.

The side effects of vaccine administration during pregnancy are shown to be the same as those seen in the general population and the efficacy is high, with an adjusted risk ratio of 0.22 . (95\% CI 0.11-0.43) ${ }^{2}$.

The crossing of the placenta by the antibodies generated through vaccination offer a level of protection that should not be ignored.

With all this information, we aimed to comparatively analyze the levels of SARS-CoV-2 IgG and IgM antibodies in pregnant women who have had the infection during pregnancy or have undergone a complete vaccination cycle during pregnancy, as well as antibody levels in newborns.

\section{MATERIAL AND METHODS}

Forty-eight women who gave birth in the Department of Obstetrics and Gynecology of the University Emergency Hospital of Bucharest between March $1^{\text {st }}, 2020$ and September 30 3021 were included in the study. The main inclusion criterion was the history of SARSCoV-2 infection during pregnancy or COVID-19 complete vaccination. For each case the peri-partum values of IgG and IgM SARS-CoV-2 antibodies were analyzed in the same laboratory along with those of their newborns. Written informed consent was obtained for each case.

This study was approved by the Ethics Committee of the University Emergency Hospital of Bucharest.

\section{RESULTS}

The vaccination rate in our study group was about $6 \%$, specifically three cases. The vaccinated patients had a significant value of protective IgG SARS-CoV-2 antibodies and even more interesting, the level of protective antibodies of the newborns closely followed maternal values (Figure 1).

As regards the cases with SARS-CoV-2 infection during pregnancy, only 8 women $(16.6 \%)$ had a protective level of antibodies at the time of testing. The greatest level was 3.46 and, for this specific case, the level of $\mathrm{IgG}$ antibodies of her child (5.03) exceeded that of the mother. Overall, $75 \%$ of the newborns of mothers with protective level of antibodies had also protective levels of IgG SARS-CoV-2 antibodies (Figure 2).

According to the results we have obtained, 4 cases had reactive levels of IgM antibodies ( $>1)$. None of their newborns had IgM positive levels, no mother in these four cases had simultaneously IgG reactive levels and only one newborn tested positive for IgG SARSCoV-2 antibodies (Figure 3). 

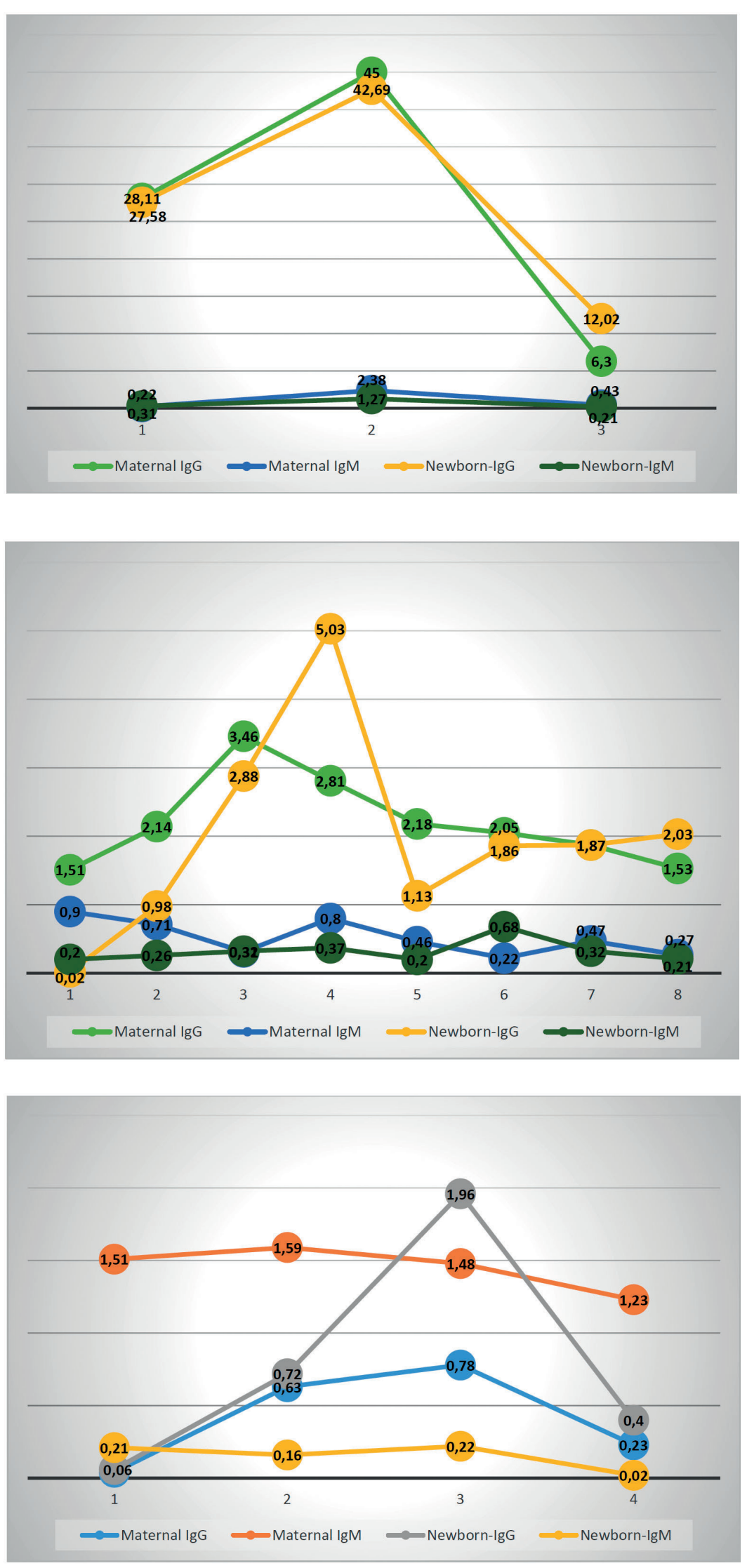

Figure 1. SARS-CoV-2 IgG and IgM levels of women completely vaccinated during pregnancy compared to the levels of their newborns

Figure 2. Comparative levels of SARS-COV-2 IgG and IgM antibodies in women with a history of COVID-19 during pregnancy and their newborns

Figure 3. Positive levels of maternal $\operatorname{IgM}$ and the corresponding level of their newborns. 


\section{DISCUSSIONS AND CONCLUSIONS}

According to the results we obtained, active but asymptomatic peri-partum SARS-CoV-2 infection was not transmitted to the infants, fact objectified by the absence of reactive IgM SARS-CoV-2 titers. It is important to mention that IgM antibodies, expressed when the infection is active, are large molecules with minimum possibility of crossing the placenta. The rate of infection transmission, reported in medical literature, is about $6 \%$, if the infection is manifested in the first and second trimester and zero, if the patient is infected in the third trimester ${ }^{6}$.

The serologic results in our study group, indicate there is a transplacental immunity obtained from mothers who have positive titers of IgG antibodies and even though the rate of vertical transmission is low; referring to immunologic action of other viruses we can presume that this protection can be active during the first period of life ${ }^{7}$.

Besides all the novelties related to SARS-CoV-2 infection prognosis, one thing is clear at this point, namely SARS-CoV-2 infection manifests with a greater severity in pregnant women compared to non-pregnant population, associating a high risk of hospital and intensive care admission and even death ${ }^{8}$. Trials regarding the safety of vaccination have begun in February 2021 and have demonstrated its safety and efficacy. The safety data available for mRNA vaccines during pregnancy have shown that maternal antibodies cross the placenta and provide neonatal protection ${ }^{9}$. According to Kathryn J. Gray et al. ${ }^{10}$, vaccine-induced antibody levels are significantly higher compared to those obtained through SARS-CoV-2 infection during pregnancy. Also, the administration of the second dose of
SARS-COV-2 vaccine results in augmented levels of $\mathrm{IgG}$ antibodies in mothers and in their newborns, offering immunologic protection. In our study group the proportion of vaccinated pregnant women was low, but the astonishing difference between the values of $\mathrm{IgG}$ antibodies in this group compared to those who have obtained natural protection strengthen the conclusion of the above-mentioned study.

As most pregnant women are reluctant to receive the SARS-CoV-2 vaccine, advice on its safety, benefits and efficacy should be clearly emphasized by specialists. One of the concerns of pregnant patients is the lack of data on the long-term effects on children born to previously vaccinated mothers, while pregnant. It is clear, as far as this issue is concerned, that the components of mRNA vaccines last in our organism only for a few days after injection as they are broken down and, in regard to this aspect alone, the pregnancy outcome of patients who received the vaccine during pregnancy is the same as it is for those who have not.

Concluding, the protective levels of antibodies after SARS-CoV-2 infection in pregnancy is low in mothers and even lower in their newborns. There is no transplacental transmission of SARS-CoV-2 IgM antibodies. Thus, our results clearly plead in favor of vaccination during pregnancy which provides significant benefits for both mothers and infants.

Compliance with ethics requirements: The authors declare no conflict of interest regarding this article. The authors declare that all the procedures and experiments of this study respect the ethical standards in the Helsinki Declaration of 1975, as revised in 2008(5) and the national law. Informed consent was obtained from the patient described in the clinical case and his parents. 


\section{References}

1. ACOG and SMFM Recommend COVID-19 Vaccination for Pregnant Individuals. https://www.acog.org/news/news-releases/2021/07/acog-smfm-recommend-covid-19-vaccination-for-pregnant-individuals

2. SMFM: Provider Considerations for Engaging in COVID-19 Vaccine Counseling with Pregnant and Lactating Patients. https:// s3.amazonaws.com/cdn.smfm.org/media/3039/Provider_Considerations_for_Engaging_in_COVID_Vaccination_Considerations_7-30-21_\%28final\%29_.pdf

3. Bwire GM, Njiro BJ, Mwakawanga DL, Sabas D, Sunguya BF. Possible vertical transmission and antibodies against SARS-CoV-2 among infants born to mothers with COVID-19: A living systematic review. J Med Virol. 2021 Mar;93(3):1361-1369.

4. Cruz-Lemini M, Ferriols Perez E, de la Cruz Conty ML, et al. Obstetric Outcomes of SARS-CoV-2 Infection in Asymptomatic Pregnant Women. Viruses. 2021 Jan 15;13(1):112.

5. COVID-19 vaccines, pregnancy and breastfeeding. Royal College of Obstetricians and Gynaecologists. https://www.rcog.org.uk/ en/guidelines-research-services/coronavirus-covid-19-pregnancy-and-womens-health/covid-19-vaccines-and-pregnancy/ covid-19-vaccines-pregnancy-and-breastfeeding/

6. Kotlyar AM, Grechukhina O, Chen A, et al. Systematic review vertical transmission of coronavirus disease 2019: a systematic review and meta-analysis [published online ahead of print July 31 , 2020]. Am J Obstet Gynecol. 2020.

7. Puck JM, Glezen WP, Frank AL, Six HR. Protection of infants from infection with influenza A virus by transplacentally acquired antibody. J Infect Dis. 1980; 142(6): 844- 849

8. Zambrano L.D, Ellington S, Strid P, et al. Update: characteristics of symptomatic women of reproductive age with laboratory-confirmed SARS-CoV-2 infection by pregnancy status - United States, January 22-October 3, 2020. MMWR Morb Mortal Wkly Rep. 2020; 69: 1641-1647

9. Centers for Disease Control and Prevention. Vaccine safety. V-safe after vaccination health checker. 2021. https://www.cdc. gov/vaccinesafety

10. Gray KJ, Bordt EA, Atyeo C, et al. Coronavirus disease 2019 vaccine response in pregnant and lactating women: a cohort study. Am J Obstet Gynecol. 2021;225(3):303.e1-303.e17. doi:10.1016/j. ajog.2021.03.023 